\title{
NORMAL AMENABLE SUBGROUPS OF THE AUTOMORPHISM GROUP OF THE FULL SHIFT
}

\author{
JOSHUA FRISCH, TOMER SCHLANK AND OMER TAMUZ
}

\begin{abstract}
We show that every normal amenable subgroup of the automorphism group of the full shift is contained in its center. This follows from the analysis of this group's Furstenberg topological boundary, through the construction of a minimal and strongly proximal action.

We extend this result to higher dimensional full shifts. This also provides a new proof of Ryan's Theorem and of the fact that these groups contain free groups.
\end{abstract}

\section{Contents}

1. Introduction

2. A boundary of $\operatorname{Aut}\left(A^{\mathbb{Z}}\right)$

2.1. Extreme proximality

2.2. Induction to a strongly proximal, minimal action 4

3. The full shift over $\mathbb{Z}$

4. The full shift over $\mathbb{Z}^{d}$

5. Construction of cellular automata 7

5.1. Defining cellular automata 7

5.2. Proof of Theorem 2.4 8

5.3. Proof of Theorem $2.1 \quad 9$

$\begin{array}{lr}\text { References } & 10\end{array}$

\section{INTRODUCTION}

For $n \geq 2$, let $A=\{0,1, \ldots, n-1\}$ be a finite alphabet. Equip the countable product $A^{\mathbb{Z}}$ with the product topology. Let $\sigma: A^{\mathbb{Z}} \rightarrow A^{\mathbb{Z}}$ be the left shift, and let $\operatorname{Aut}\left(A^{\mathbb{Z}}\right)$ be the group of homeomorphisms of $A^{\mathbb{Z}}$ that commute with the shift $\sigma$. The space $A^{\mathbb{Z}}$ is called the full shift, and $\operatorname{Aut}\left(A^{\mathbb{Z}}\right)$ is called the automorphism group of the full shift. The elements of this group are known as (invertible) cellular automata.

Date: June 28, 2017.

This research was partially conducted at Microsoft Research, New England. 
This group has been studied extensively, starting with Hedlund [10], who showed that it is countable, and that in many senses it very large; in particular, it contains free groups of every rank, and hence it is non-amenable ${ }^{1}$. Ryan [13] showed that its center $Z\left(\operatorname{Aut}\left(A^{\mathbb{Z}}\right)\right)$ is equal to $\Sigma\left(A^{\mathbb{Z}}\right)$, the group consisting of the powers of the shift $\sigma$. Boyle, Lind and Rudolph [1] made further progress, extended many results to automorphism groups of shifts of finite type, and noted that $\operatorname{Aut}\left(2^{\mathbb{Z}}\right)$ and $\operatorname{Aut}\left(4^{\mathbb{Z}}\right)$ are not algebraically isomorphic ${ }^{2}$.

Our main theorem is a strengthening of Ryan's:

Theorem 1.1. Every normal amenable subgroup of $\operatorname{Aut}\left(A^{\mathbb{Z}}\right)$ is contained in $\Sigma\left(A^{\mathbb{Z}}\right)$.

For every group $G$ there exists a maximal normal amenable subgroup called the amenable radical (see for example [12]); we denote it by $\sqrt{G}$. Thus this theorem in fact states that $\sqrt{\operatorname{Aut}\left(A^{\mathbb{Z}}\right)}=\Sigma\left(A^{\mathbb{Z}}\right)$.

Furman [7] showed that the amenable radical is the kernel of a group's action on its Furstenberg topological boundary. A topological boundary of a group $G$ is a compact $G$-space $X$ such that the continuous $G$ action on $X$ is minimal and strongly proximal $[8,9]$. The Furstenberg topological boundary $B(G)$ (or the maximal boundary) is the universal topological boundary, in the sense that it admits a $G$ equivariant map to any $G$-boundary. Since $Z(G) \subseteq \sqrt{G}$, and since the extension of a faithful action is faithful, it follows that

Theorem 1.2 (Furman [7]). If a group $G$ has a topological boundary $X$ on which the action of $G / Z(G)$ is faithful, then $\sqrt{G}=Z(G)$.

To prove our main result, Theorem 1.1, we construct a topological boundary of $\operatorname{Aut}\left(A^{\mathbb{Z}}\right)$ whose kernel is equal to $\Sigma\left(A^{\mathbb{Z}}\right)$; our theorem then follows from Furman's. To show that our action is strongly proximal we use Glasner's notion of an extremely proximal action [9]. We define these terms precisely in the next section.

One can replace $\mathbb{Z}$ with $\mathbb{Z}^{d}$ in the discussion above. In this case the shift $\sigma$ and the group it generates are replaced with the $d$ shifts which generate $\Sigma\left(A^{\mathbb{Z}^{d}}\right)$. The automorphism group $\operatorname{Aut}\left(A^{\mathbb{Z}^{d}}\right)$ is defined to be group of homeomorphisms of $A^{\mathbb{Z}^{d}}$ that commutes with $\Sigma\left(A^{\mathbb{Z}^{d}}\right)$.

Hochman [11] proves in this setting an analogue of Ryan's Theorem, namely that $Z\left(\operatorname{Aut}\left(A^{\mathbb{Z}^{d}}\right)\right)=\Sigma\left(A^{\mathbb{Z}^{d}}\right)$, and in fact shows that the same holds for the automorphism groups of a large class of shifts. We likewise strengthen his theorem, for the case of the full shift.

\footnotetext{
${ }^{1}$ Hedlund attributes most of these results to Curtis, Lyndon and Hedlund.

${ }^{2}$ It is not known if $\operatorname{Aut}\left(2^{\mathbb{Z}}\right)$ and $\operatorname{Aut}\left(3^{\mathbb{Z}}\right)$ are isomorphic.
} 
Theorem 1.3. Every normal amenable subgroup of $\operatorname{Aut}\left(A^{\mathbb{Z}^{d}}\right)$ is contained in $\Sigma\left(A^{\mathbb{Z}^{d}}\right)$.

Recently, Cyr and Kra [4] showed that some subshifts ${ }^{3}$ of $A^{\mathbb{Z}}$ with sub-exponential growth have amenable automorphism groups. Their work follows a number of papers that show that the automorphism group of "small" shifts is indeed "small" $[2,3,5,6,14,15]$. A natural question is the following: for which subshifts of $A^{\mathbb{Z}}$ does it still hold that the amenable radical of the automorphism group is equal to its center?

\section{A boundary of $\operatorname{Aut}\left(A^{\mathbb{Z}}\right)$}

In this section we denote $G=\operatorname{Aut}\left(A^{\mathbb{Z}}\right)$ and $\Sigma=\Sigma\left(A^{\mathbb{Z}}\right)$.

Let $A_{p}^{\mathbb{Z}} \subset A^{\mathbb{Z}}$ be the set of configurations (as we shall refer to elements of $A^{\mathbb{Z}}$ ) that have a constant infinite prefix:

$$
A_{p}^{\mathbb{Z}}=\left\{x \in A^{\mathbb{Z}}: \exists m \in \mathbb{Z}, a \in A \text { s.t. } x_{k}=a \text { for all } k \leq m\right\} .
$$

Note that this set is invariant to the $G$-action.

Let $A_{*}^{\mathbb{Z}} \subset A_{p}^{\mathbb{Z}}$ be the result of the exclusion from $A_{p}^{\mathbb{Z}}$ of the $n$ constant configurations. This set is still $G$-invariant. Given $x \in A_{*}^{\mathbb{Z}}$, let $\ell(x)$ denote the last coordinate of the constant prefix:

$$
\ell(x)=\min \left\{m \in Z: x_{m} \neq x_{m+1}\right\} .
$$

Note that $\ell(\sigma x)=\ell(x)-1$, in general $\ell\left(\sigma^{k} x\right)=\ell(x)-k$, and in particular $\ell\left(\sigma^{\ell(x)} x\right)=0$.

Let $\Omega$ be given by

$$
\Omega=\left\{x:\{0,1,2, \ldots\} \rightarrow A: x_{0} \neq x_{1}\right\} .
$$

This is the space of one-sided infinite configurations, in which the zeroth symbol is different than the first. We equip it with the natural topology induced from the product topology.

To define a $G$ action on $\Omega$, let $\varphi: \Omega \rightarrow A_{*}^{\mathbb{Z}}$ assign to $x \in \Omega$ the twosided configuration in which a one-sided, infinite constant $x_{0}$ prefix precedes $x_{1} x_{2} \ldots$ Formally:

$$
[\varphi(x)]_{m}=\left\{\begin{array}{ll}
x_{0} & m \leq 0 \\
x_{m} & \text { otherwise }
\end{array} .\right.
$$

Note that the image of $\varphi$ is all the configurations in $A_{*}^{\mathbb{Z}}$ for which $\ell(x)=$ 0 . Hence $\varphi^{-1}\left(\sigma^{\ell(x)} x\right)$ is well defined for every $x \in A_{*}^{\mathbb{Z}}$. Accordingly,

\footnotetext{
${ }^{3} \mathrm{~A}$ shift or subshift of $A^{\mathbb{Z}}$ is a closed, shift-invariant subset of $A^{\mathbb{Z}}$.
} 
define $\psi: A_{*}^{\mathbb{Z}} \rightarrow \Omega$ by $\psi(x)=\varphi^{-1}\left(\sigma^{\ell(x)} x\right)$; note that $\psi \circ \varphi$ is the identity. Now, given $g \in G$ and $x \in \Omega$ let

$$
g x=\psi(g \varphi(x)) .
$$

It is straightforward to verify that this is indeed a $G$-action on $\Omega$. Note also that this action factors through $G / \Sigma$, since $\sigma f=\sigma$ for all $x \in \Omega$. Additionally, it is easy to see that the $G / \Sigma$-action is faithful; this is due to the fact that $\psi^{-1}(\Omega)$ is dense in $A^{\mathbb{Z}}$.

For $a \in A$, let $\Omega^{a}=\left\{x \in \Omega: x_{0}=a\right\}$. Let $G^{a} \subset G$ be the finite index subgroup that fixes $\Omega^{a}$, and let $G^{*}=\cap_{a} G^{a}$.

2.1. Extreme proximality. An action $H \curvearrowright X$ of a discrete group on a compact metric space is said to be extremely proximal if, for any closed $Y \subsetneq X$, there exists a sequence $\left\{h_{k}\right\} \subset H$ such that $\lim _{k} h_{k} Y$ is a singleton, where the limit is taken in the Hausdorff topology [9].

An action $H \curvearrowright X$ of a discrete group on a compact Hausdorff space is said to be strongly proximal if, for any Borel probability measure $\mu$ on $X$, there exists a sequence $\left\{h_{k}\right\} \subset H$ such that $\lim _{k} h_{k} \mu$ is a point mass, where the limit is taken in the weak* topology $[8,9]$. We prove the following theorem in Section 5.

Theorem 2.1. The action $G^{*} \curvearrowright \Omega^{a}$ is extremely proximal for all $a \in A$.

We can now conclude that $G^{*}$ is not amenable, and in fact includes a free group with two generators. This follows from the following theorem.

Theorem 2.2 (Glasner [9]). If a group has a non-trivial minimal extremely proximal action then it contains a free subgroup on two generators.

The fact that $G^{*}$ is contains a free subgroup on two generators was already shown in [10], and thus this provides a new proof of that fact.

2.2. Induction to a strongly proximal, minimal action. Recall that $\Omega^{a}$ is the subset of all $x \in \Omega$ such that $x_{0}=a$. Let $\bar{\Omega}$ be the collection of subsets of $\Omega$ which intersect each $\Omega^{a}$ in exactly one element. Hence every element of $\bar{\Omega}$ is a set of size $n=|A|$, and can be written as

$$
\left\{x^{0}, x^{1}, \ldots, x^{n-1}\right\}
$$

with $x_{0}^{a}=a$ for all $a \in A$. The topology on $\bar{\Omega}$ is inherited from $\Omega$ in the obvious way.

There is a natural $G$ action on $\bar{\Omega}$, derived from the action on $\Omega$, and hence on the subsets of $\Omega$. 
Since the elements of $G^{*}$ preserve $x_{0}$ - that is, $[g x]_{0}=x_{0}$ for all $x \in \Omega$ and $g \in G^{*}$ - the action of $G^{*}$ on $\bar{\Omega}$ is isomorphic to the diagonal action $G^{*} \curvearrowright \Omega^{0} \times \Omega^{1} \times \cdots \times \Omega^{n-1}$. Since each action $G^{*} \curvearrowright \Omega^{a}$ is extremely proximal (Theorem 2.1), the product action $G^{*} \curvearrowright \Omega^{0} \times$ $\Omega^{1} \times \cdots \times \Omega^{n-1}$ is strongly proximal. This follows from the facts that (i) extremely proximal actions are strongly proximal and (ii) that a product of strongly proximal actions is likewise strongly proximal [9]. Hence we have shown the following theorem.

Theorem 2.3. The $G^{*}$ action on $\bar{\Omega}$ is strongly proximal.

We next show that this action is also minimal.

Theorem 2.4. The $G^{*}$ actions on both $\bar{\Omega}$ and $\Omega^{0}$ are minimal.

We prove this theorem in Section 5.

\section{The FUlL SHIFT OVER $\mathbb{Z}$}

Given the construction of the previous section, the proof of our main theorem is immediate.

Proof of Theorem 1.1. Note that $\bar{\Omega}$ is a topological boundary of $\operatorname{Aut}\left(A^{\mathbb{Z}}\right)$, since it is strongly proximal (Theorem 2.3) and minimal (Theorem 2.4). Since this action is a faithful action of $\operatorname{Aut}\left(A^{\mathbb{Z}}\right) / \Sigma\left(A^{\mathbb{Z}}\right)$, the claim follows by Theorem 1.2.

\section{The FULL SHIFT OVER $\mathbb{Z}^{d}$}

In this section we extend our result to show that the amenable radical of $\operatorname{Aut}\left(A^{\mathbb{Z}^{d}}\right)$ is the group of shifts. We do this by essentially reducing the higher dimensional case to the one dimensional case.

Fix a dimension $d$. For $k \in \mathbb{N}$, let $M_{k}$ be the basis for $\mathbb{Z}^{d}$ given by the rows of the following matrix:

$$
\left(\begin{array}{cccccc}
1 & k & 0 & \cdots & 0 & 0 \\
0 & 1 & k & \cdots & 0 & 0 \\
\vdots & \vdots & \vdots & \ddots & \vdots & \vdots \\
0 & 0 & 0 & \cdots & 1 & k \\
0 & 0 & 0 & \cdots & 0 & 1
\end{array}\right) .
$$

Let $\mathbf{v}$ be the unit vector of the $d^{\text {th }}$ coordinate (which is also the last vector in $M_{k}$ ), and let $U_{k} \subset \mathbb{Z}^{d}$ the span of the first $d-1$ vectors in $M_{k}$. Then every element of $\mathbb{Z}^{d}$ can be uniquely written as $\mathbf{u}+\ell \cdot \mathbf{v}$ where $\mathbf{u} \in U_{k}$ and $\ell \in \mathbb{Z}$.

The important property of $U_{k}$ is the following. 
Claim 4.1. Every nonzero element $\mathbf{u} \in U_{k}$ has norm greater than $k$.

Proof. Represent $\mathbf{u}$ as an integral linear combination of the first $d-1$ vectors in $M_{k}$, and note that for $\mathbf{u}$ to be nonzero there must be a largest index $i$ such that the coefficient given by the $i^{\text {th }}$ basis vector is nonzero. This implies that the $i+1^{\text {st }}$ coordinate is a nonzero multiple of $k$, which implies that the norm of $\mathbf{u}$ is at least $k$.

Let $A_{U_{k}}^{\mathbb{Z}^{d}}$ be the subset of $A^{\mathbb{Z}^{d}}$ which is periodic mod $U_{k}$; that is, under the natural shift action of $\mathbb{Z}^{d}$ on $A^{\mathbb{Z}^{d}}, A_{U_{k}}^{\mathbb{Z}^{d}}$ is the set of $U_{k}$-invariant elements of $A^{\mathbb{Z}^{d}}$. Note that $A_{U_{k}}^{\mathbb{Z}^{d}}$ is a closed subset of $A^{\mathbb{Z}^{d}}$. We endow it with the induced topology. After proving a simple claim, we will proceed to show how $A_{U_{k}}^{\mathbb{Z}^{d}}$ can be identified with $A^{\mathbb{Z}}$.

The next claim follows directly from the definition of $A_{U_{k}}^{\mathbb{Z}^{d}}$ and Claim 4.1.

Claim 4.2. The projection of $A_{U_{k}}^{\mathbb{Z}^{d}}$ to $B_{k}$, the ball of radius $k$ in $\mathbb{Z}^{d}$, is equal to $A^{B_{k}}$.

That is, any $x \in A^{B_{k}}$ can be completed to an element of $A_{U_{k}}^{\mathbb{Z}^{d}}$.

Using the obvious group isomorphism between $\mathbb{Z}$ and $\mathbb{Z} \cdot \mathbf{v} \subset \mathbb{Z}^{d}$ (recall that $\mathbf{v}$ is the last vector in $M_{k}$ ), we obtain the homeomorphism $\pi: A_{U_{k}}^{\mathbb{Z}^{d}} \rightarrow A^{\mathbb{Z}}$ given by

$$
[\pi(x)]_{n}=x_{n \cdot \mathbf{v}} .
$$

Note that this is indeed a bijection since $\mathbb{Z} \cdot \mathbf{v}$ is a set of representatives $\mathbb{Z}^{d} / U_{k}$, the cosets of $U_{k}$ in $\mathbb{Z}^{d}$. It is straightforward to check that $\pi$ is also continuous.

Accordingly, we define a group homomorphism $\phi_{k}: \operatorname{Aut}\left(A^{\mathbb{Z}^{d}}\right) \rightarrow$ $\operatorname{Aut}\left(A^{\mathbb{Z}}\right)$ by setting $\phi_{k}(g)=\pi \circ g \circ \pi^{-1}$. Note that $\pi^{-1} \circ \sigma \circ \pi$, the conjugation of the shift on $A^{\mathbb{Z}}$ by $\pi$, is a shift on $A_{U_{k}}^{\mathbb{Z}^{d}}$. It follows that $\phi_{k}(g)$ commutes with the shift $\sigma$, and hence the image of $\phi_{k}$ is indeed in $\operatorname{Aut}(\mathbb{Z})$.

Let $L_{r} \subset \operatorname{Aut}\left(A^{\mathbb{Z}^{d}}\right)$ be the set of cellular automata with memory less than $r$. That is, $g \in L_{r}$ if $[g(x)]_{0}$ is determined by the restriction of $x$ to some ball of radius less than $r$ around 0 .

Claim 4.3. If $g \in L_{k}$ then $g$ is the unique element in $\phi_{k}^{-1}(g) \cap L_{k}$.

Proof. Every $g \in L_{k}$ is uniquely determined, among elements of $L_{k}$, by its action on $A_{U_{k}}^{\mathbb{Z}^{d}}$; this follows from Claim 4.2. Since the kernel of $\phi_{k}$ is the kernel of the action $\operatorname{Aut}\left(A^{\mathbb{Z}^{d}}\right) \curvearrowright A_{U_{k}}^{\mathbb{Z}^{d}}$, it follows that if $g$ has memory less than $k$ then $g$ is the unique element in $L_{k}$ that is mapped to $\phi(g)$. 
Note that there may, however, be other elements of $\operatorname{Aut}\left(A^{\mathbb{Z}^{d}}\right)$, which will not be in $L_{k}$, whose action on $A_{U_{k}}^{\mathbb{Z}^{d}}$ is the same as that of $g$.

We will exploit these homomorphisms $\phi_{k}$ for varying $k$ in order to prove our theorem. We first note the following easy lemma.

Lemma 4.4. Let $\phi: H \rightarrow K$ be a group homomorphism, and let $\sqrt{H}$ denote the amenable radical of $H$. Then $\sqrt{H} \subseteq \phi^{-1}(\sqrt{K})$.

Proof. This follows immediately from the fact that both amenable groups and normal subgroups are preserved under quotients.

We are now in a position to prove the main theorem of this section.

Proof of Theorem 1.3. Choose $g \in \sqrt{\operatorname{Aut}\left(A^{\mathbb{Z}^{d}}\right)}$. Then $g$ has memory less than $k$ for some large enough $k$, i.e., $g \in L_{k}$. From 4.4 it follows that $\phi_{k}(g) \in \sqrt{\operatorname{Aut}(\mathbb{Z})}$, and so $\phi_{k}(g)$ is a shift $\sigma^{m}$, for some $m \in \mathbb{Z}$, by Theorem 1.1.

We now claim that since $\phi_{k}(g)$ is a shift and since $g \in L_{k}$, then $g$ is a shift. Showing this will conclude the proof that the amenable radical of $\operatorname{Aut}\left(A^{\mathbb{Z}^{d}}\right)$ is equal to the shifts. To see this, note that since $\phi_{k}(g)=\sigma^{m}$ then for every $x \in A^{\mathbb{Z}},\left[\phi_{k}(g) x\right]_{0}=x_{m}$. Hence, by the definition of $\phi_{k}$, for every $y \in A_{U_{k}}^{\mathbb{Z}^{d}}$ it holds that $[g y]_{\mathbf{0}}=y_{m \cdot \mathbf{v}}$. By the definition of $A_{U_{k}}^{\mathbb{Z}^{d}}$, $y_{m \cdot \mathbf{v}}=y_{m \cdot \mathbf{v}+\mathbf{u}}$ for every $\mathbf{u} \in U_{k}$. Since $g \in L_{k}$, it follows that the norm of $m \cdot \mathbf{v}+\mathbf{u}$ is at most $k$ for some $\mathbf{u}$. Therefore the shift by $m \cdot \mathbf{v}+\mathbf{u}$ is also in $L_{k}$. But by Claim 4.3 the unique element that is both in $L_{k}$ and $\phi^{-1}(g)$ is $g$, and hence $g$ is the shift by $m \cdot \mathbf{v}+\mathbf{u}$.

\section{Construction of Cellular automata}

5.1. Defining cellular automata. To define invertible cellular automata on $A^{\mathbb{Z}}$ we will use the following general scheme ${ }^{4}$. First, we fix a start marker $S$ and an end marker $E$, where $S \in A^{k}$ and $E \in A^{k^{\prime}}$ for some $k, k^{\prime} \in \mathbb{N}$. We choose an $n \in \mathbb{N}$ and call some subset $\mathcal{D} \subseteq A^{n}$ the set of possible data. Finally, we let $\pi$ be a bijection $\pi: \mathcal{D} \rightarrow \mathcal{D}$. In our constructions, $\pi$ will always be an involution. We then define a cellular automaton $g: A^{\mathbb{Z}} \rightarrow A^{\mathbb{Z}}$ by the mapping $S D E \rightarrow S \pi(D) E$, where the $\operatorname{data} D$ is an element of $\mathcal{D}$. That is, let $x \in A^{\mathbb{Z}}$, and denote $x_{m, m^{\prime}}=x_{m} x_{m+1} \ldots x_{m^{\prime}-1}$. Then if $x_{m, m+k+n+k^{\prime}}=S D E$ for some $D \in \mathcal{D}$ then $[g(x)]_{m, m+k+n+k^{\prime}}=S \pi(D) E$, and everywhere else $g$ is the identity. We will use the following notation to define particular automata. For example, if $S=000, E=111, \mathcal{D}=\{2332,3223\}$ and

\footnotetext{
${ }^{4}$ This scheme is a generalization of the one used in [1, Section 2].
} 
$\pi(2332)=(3223)$ then we will define $g$ by the diagram below, in which the data appear in boldface.

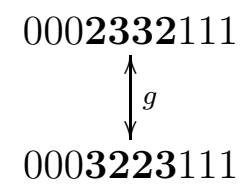

For such an automaton to be well defined, it suffices to show that no two data matches overlap; that is, if $x_{m, m+k+n+k^{\prime}}=S D E$ and $x_{m^{\prime}, m^{\prime}+k+n+k^{\prime}}=S D^{\prime} E$ for some $m \neq m^{\prime}$ and $D, D^{\prime} \in \mathcal{D}$, then the data match intervals $[m+k, m+k+n)$ and $\left[m^{\prime}+k, m^{\prime}+k+n\right)$ do not intersect. To show that such an automaton is invertible, it suffices to furthermore show that no data match overlaps a marker match. That is, the data match interval $[m+k, m+k+n)$ does not intersect either of the marker match intervals $\left[m^{\prime}, m^{\prime}+k\right)$ and $\left[m^{\prime}+k+n, m^{\prime}+k+n+k^{\prime}\right)$. We refer to these conditions below as the overlap conditions.

We will need to slightly generalize this construction to cellular automata where there is a collection of start markers $\left(S_{1}, \ldots, S_{\ell}\right)$, corresponding end markers $\left(E_{1}, \ldots, E_{\ell}\right)$ corresponding data sets $\left(\mathcal{D}_{1}, \ldots, \mathcal{D}_{\ell}\right)$ and corresponding bijections $\left(\pi_{1}, \ldots, \pi_{\ell}\right)$. As before, if $x_{m, m+k+n+k^{\prime}}=$ $S_{i} D E_{i}$ for some $D \in \mathcal{D}_{i}$ then $[g(x)]_{m, m+k+n+k^{\prime}}=S_{i} \pi_{i}(D) E_{i}$. To ensure well-defindedness and invertibility, similar overlap conditions need to apply. That is, that no data match overlaps another data or marker match, whether of the same index $i$ or not. To specify such automata we will use similar diagrams, for example

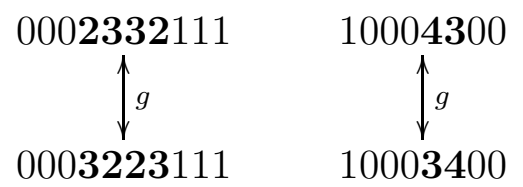

Note that in this example the markers do overlap, but the data cannot.

\subsection{Proof of Theorem 2.4.}

Proof of Theorem 2.4. We show that the $G^{*}$ action on $\bar{\Omega}$ is minimal. The proof that the action on $\Omega^{0}$ is minimal follows by the same argument.

To this end, we choose arbitrary $\bar{\omega}, \bar{\eta} \in \bar{\Omega}$ and show that there exists a sequence $g_{k} \in G^{*}$ such that $\lim _{k} g_{k} \bar{\omega}=\bar{\eta}$.

For each $a \in A$, define $x^{a}, y^{a} \in \Omega^{a}$ by $\bar{\omega} \cap \Omega^{a}=\left\{x^{a}\right\}$ and $\bar{\eta} \cap \Omega^{a}=$ $\left\{y^{a}\right\}$. Denote $x_{[k]}^{a}=x_{1}^{a} \ldots x_{k}^{a} \in A^{k}$ and likewise $y_{[k]}^{a}=y_{1}^{a} \ldots y_{k}^{a} \in A^{k}$. 
Define the transformation $g_{k}$ as follows.

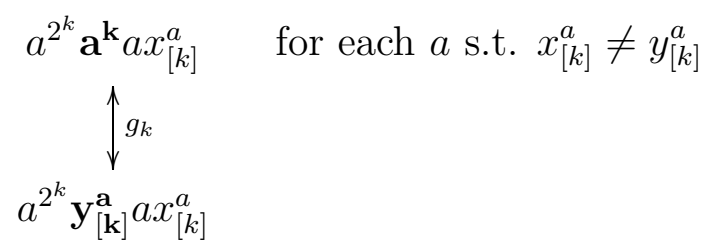

Note that the choice of $2^{k}$ in the start marker is somewhat arbitrary; we could have chosen any function that is sufficiently larger than $k$.

Note also that $x_{1}^{a}$ and $y_{1}^{a}$ are both not equal to $a$. Using this, and that fact that the end marker starts with $a$, it is straightforward (if tedious) to verify that the overlap conditions of Section 5.1 are satisfied.

Finally, it is likewise easy to see that $\lim _{k} g_{k} x^{a}=y^{a}$ in particular, $\left[g_{k}\left(x^{a}\right)\right]_{[k]}=y_{[k]}$. Hence $\lim _{k} g_{k} \bar{\omega}=\bar{\eta}$.

5.3. Proof of Theorem 2.1. Let $o \in \Omega^{0}$ be given by $o_{0}=0$ and $o_{m}=1$ for all $m>0$. Given $f \in \Omega^{0}$, let $r(f)$ measure the length of the initial sequence of ones in $f$ :

$$
r(f)=\min \left\{m \geq 0: f_{m+1} \neq 1\right\} .
$$

Note that $r(f)$ is well defined for $f \in \Omega^{0}$ except $o$; we define $r(o)=\infty$. For $m \in \mathbb{N}$ define

$$
C_{m}=r^{-1}(m)=\left\{f \in \Omega^{0}: r(f)=m\right\} .
$$

Note that $\cup_{m=1}^{\infty} C_{m}=\Omega^{0} \backslash\{o\}$, that each $C_{m}$ is closed, and that $\lim _{m} C_{m}=\{o\}$ for all $m$.

We now define a sequence $\left\{g_{k}\right\}_{k>0} \subset G^{*}$ as follows:

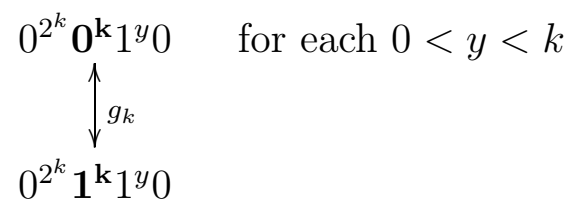

If $|A| \geq 3$, then in addition we let $a$ be any symbol in $A$ that does not equal 0 or 1 , and add to $g_{k}$ the following transformations:

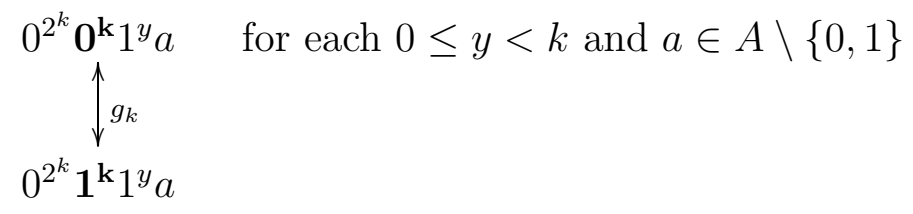


For example, two transformations performed by $g_{3}$ are
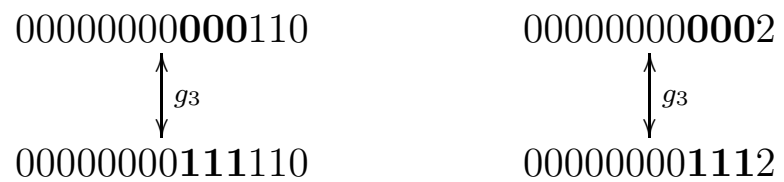

Using the fact that $y$ is strictly less than $k$, it is straightforward to check the overlap conditions of Section 5.1, and hence each $g_{k}$ is a well defined involution.

Now, note that if $r(f)<k$ then $r\left(g_{k} f\right)=r(f)+k$. Hence, for all $k>m$ we have that $g_{k} C_{m} \subseteq C_{m+k}$. Hence

Claim 5.1. $\lim _{k} g_{k} C_{m}=\lim _{k} C_{m+k}=\{o\}$.

Proof of Theorem 2.1. Without loss of generality, assume $a=0$. Let $C \subsetneq \Omega^{0}$ be closed. Then there exists a $g_{0} \in G^{*}$ such that $g_{0} C$ does not include $o$, by the minimality of the $G^{*}$ action on $\Omega^{0}$ (Theorem 2.4). Since $g_{0} C$ is closed, it is disjoint from some neighborhood of $o$, and so it is contained in the finite union $\cup_{i=1}^{m} C_{i}$, for some $m$ large enough. Let $\bar{g}_{k}=g_{k} g_{0}$, where $g_{k}$ is as defined above. Then

$$
\lim _{k} \bar{g}_{k} C=\lim _{k} g_{k} g_{0} C \subseteq \lim _{k} g_{k} \cup_{i=1}^{m} C_{i}=\cup_{i=1}^{m} \lim _{k} g_{k} C_{i}=\{o\},
$$

where the last equality follows from Claim 5.1. But the first limit cannot be an empty set, and so

$$
\lim _{k} \bar{g}_{k} C=\{o\} .
$$

\section{REFERENCES}

[1] Mike Boyle, Douglas Lind, and Daniel Rudolph, The automorphism group of a shift of finite type, Transactions of the American Mathematical Society 306 (1988), no. 1, 71-114.

[2] Ethan Coven, Anthony Quas, and Reem Yassawi, Automorphisms of some toeplitz and other minimal shifts with sublinear complexity, arXiv preprint arXiv:1505.02482 (2015).

[3] Van Cyr and Bryna Kra, The automorphism group of a shift of subquadratic growth, arXiv preprint arXiv:1403.0238 (2014).

[4] - The automorphism group of a minimal shift of stretched exponential growth, arXiv preprint arXiv:1509.08493 (2015).

[5] _ The automorphism group of a shift of linear growth: beyond transitivity, Forum of mathematics, sigma, 2015, pp. e5.

[6] Sebastián Donoso, Fabien Durand, Alejandro Maass, and Samuel Petite, On automorphism groups of low complexity minimal subshifts, arXiv preprint arXiv:1501.00510 (2015).

[7] Alex Furman, On minimal, strongly proximal actions of locally compact groups, Israel Journal of Mathematics 136 (2003), no. 1, 173-187. 
[8] Harry Furstenberg, A poisson formula for semi-simple lie groups, Annals of Mathematics (1963), 335-386.

[9] Shmuel Glasner, Topological dynamics and group theory, Transactions of the American Mathematical Society 187 (1974), 327-334.

[10] Gustav A. Hedlund, Endomorphisms and automorphisms of the shift dynamical system, Mathematical systems theory 3 (1969), no. 4, 320-375.

[11] Michael Hochman, On the automorphism groups of multidimensional shifts of finite type, Ergodic Theory and Dynamical Systems 30 (2010), no. 03, 809840.

[12] Amos Nevo, Boundary theory and harmonic analysis on boundary transitive graphs, American Journal of Mathematics 116 (1994), no. 2, pp. 243-282.

[13] J.Patrick Ryan, The shift and commutativity, Mathematical systems theory 6 (1972), 82-85.

[14] Ville Salo, Toeplitz subshift whose automorphism group is not finitely generated, arXiv preprint arXiv:1411.3299 (2014).

[15] Ville Salo and Ilkka Törmä, Block maps between primitive uniform and pisot substitutions, Ergodic Theory and Dynamical Systems (2014), 1-19.

(J. Frisch, O. Tamuz) California Institute of Technology.

(T. Schlank) HeBrew University. 